\title{
Strategies for measuring fuel rho-R and mix using gamma-rays
}

L. A. Bernstein, J. A. Caggiano, C. Cerjan, R. Fortner, H. Herrmann, N. Hoffman, R. W. Hoffman, Y. Kim, A. Kritcher, D. H. Schneider, D. Shaughnessy, W. Stoeffl, K. J. Moody

October 4, 2010 
This document was prepared as an account of work sponsored by an agency of the United States government. Neither the United States government nor Lawrence Livermore National Security, LLC, nor any of their employees makes any warranty, expressed or implied, or assumes any legal liability or responsibility for the accuracy, completeness, or usefulness of any information, apparatus, product, or process disclosed, or represents that its use would not infringe privately owned rights. Reference herein to any specific commercial product, process, or service by trade name, trademark, manufacturer, or otherwise does not necessarily constitute or imply its endorsement, recommendation, or favoring by the United States government or Lawrence Livermore National Security, LLC. The views and opinions of authors expressed herein do not necessarily state or reflect those of the United States government or Lawrence Livermore National Security, LLC, and shall not be used for advertising or product endorsement purposes.

This work performed under the auspices of the U.S. Department of Energy by Lawrence Livermore National Laboratory under Contract DE-AC52-07NA27344. 


\title{
Deliverable for the Systems Integration Working Group Strategies for measuring fuel $\rho R$ and mix using $\gamma$-rays
}

Task I: An evaluation of the determination of $\rho R_{\text {fuel }}$ using $\mathrm{t}>250$ ps $\gamma$-rays observed using GRH arising from the capture of low energy neutrons $(<2$ $\mathrm{MeV}$ ) in the $\mathrm{Au}$ hohlraum

Task II: Documentation of potential late-time $\gamma$-ray based mix measurements arising from $(\alpha, n \gamma)$ reactions in the hot spot

Task III: Assessment of $\gamma$-ray production nuclear data used in code databases

L.A. Bernstein, J.A. Caggiano, C. Cerjan, R. Fortner, H. Herrmann, N. Hoffman, R.W. Hoffman, Y. Kim, A. Kritcher, D.H.G. Schneider, D. Shaughnessy, W. Stoeffl, K.J. Moody

\begin{abstract}
In this paper we will describe several $\gamma$-ray based approaches for determining quantities relevant to achieving ignition at NIF: $\rho R_{\text {fuel }}$ and hot spot-ablator mix. These approaches use the Gamma Reaction History (GRH) diagnostic and another potential quickly designed $\gamma$-ray detector. In addition, a preliminary partial evaluation of the quality of the existing $\gamma$-ray production nuclear databases used in modeling and interpreting the data for the documented approaches.
\end{abstract}

\section{Introduction}

Prompt and late-time $\gamma$-rays, defined in this paper as photons from nuclear processes, provide a unique window into the performance of inertial confinement fusion (ICF) capsules during peak compression conditions when the majority of nuclear processes occur. A number of these capabilities have already been recognized by the ICF community, and a Gas Cerenkov Detector-based Gamma Reaction History (GRH) is being implemented at the National Ignition Facility (NIF) to determine "gamma bang time" and provide insight into the $\rho R$ of plastic ablator material remaining at peak compression. In this paper we will explore whether GRH can also be used to a) determine the $\rho R$ of the cold fuel and, b) quantify the amount ablator material mixed into the central hot spot of the capsule. These concepts presented in this paper are preliminary and require additional validation in the form of both advanced simulations and experimental calibration for proper development.

GRH is comprised of threshold detectors that are capable of observing $\gamma$-rays with energies as low as $2.9 \mathrm{MeV}$. This threshold removes most background from photons produced through laser-plasma interactions. However, many $\gamma$-rays from the decay of low-lying isomeric nuclear states could potentially be used as diagnostics for NIF. In this paper we will mention a potential isomeric nuclear $\gamma$-ray that could be used to 
measure mix of tracer nuclides implanted into the inner layer of the ablator into the hot spot. We will also discuss the general characteristics of a detector designed to measure these isomeric $\gamma$-rays.

Lastly, all of these diagnostics rely on nuclear data from existing nuclear databases for the production of prompt and late-time $\gamma$-rays from neutron- and charged-particle induced reactions. At the end of this report we will point to some recent experimental results that indicate the uncertainties of these data could be on the order of $\pm 50 \%$.

\section{Task I: An evaluation of the utility of $t>250$ ps hohlraum $\gamma$-rays for measuring $\rho R_{\text {fuel }}$}

Recent simulation indicate that low- to moderate-yield capsules contain significant quantities of low energy $\left(E_{n}<1 \mathrm{MeV}\right)$ neutrons due to downscatter of primary neutrons in the cold dense fuel. These low-energy neutrons emerge from the NIF capsule significantly later than primary neutrons from D+T (14 MeV), D+D (2.45 MeV) or T+T $(<9 \mathrm{MeV})$ reactions. These low energy neutrons are highly likely to be captured in the Gold hohlraum, thereby producing a significant number of high-energy $\left(E_{\mathrm{g}}>4 \mathrm{MeV}\right)$ "late-time" ( $\geq 250 \mathrm{ps}) \gamma$-rays. In this paper we will consider whether these high-energy photons will produce signals in the Gamma Reaction History (GRH) diagnostic that can be used to determine fuel areal density $(\rho R)$ in a plastic NIF capsules.

Figure 1 below shows neutron spectra from simulations of a wide range of NIF capsule.

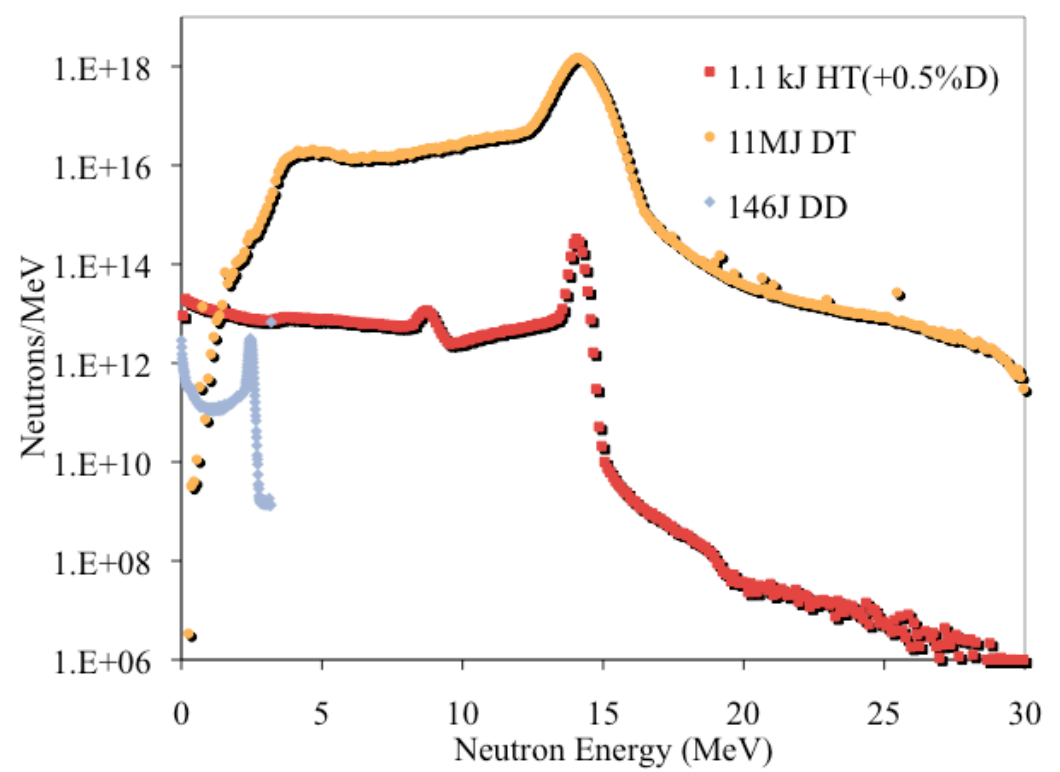

Figure 1: Simulated neutron spectra from an $11 \mathrm{MJ}$ DT (gold), $1.1 \mathrm{~kJ}$ $\mathrm{HT}(+0.5 \% \mathrm{D})$ (red), and $146 \mathrm{~J}$ pure D (gray) cryogenic indirect drive NIF capsules. Note the large number of low energy neutrons in both the HTD and DD cases. 
The high yield DT shot contains few low-energy $(<2 \mathrm{MeV})$ neutrons since the rapid evolution of energy in the capsule causes it to disassemble quickly thereby not allowing the multiple neutron scattering needed to "thermalize". In contrast, both the 146 J DD and $1.1 \mathrm{~kJ} \mathrm{HT}(0.5 \% \mathrm{D})$ shots, which remain assembled far longer, show a large number of low energy neutrons from multiple scatters of primary neutrons in the cold dense fuel.

Low energy neutrons can generate $\gamma$-rays via two processes: $\left(n, n^{\prime} \gamma\right)$ and $(n, \gamma)$. While $(\mathrm{n}, \mathrm{n}$ ' $\gamma)$ can only produce $\gamma$-rays with energies less than the incident neutron, $(\mathrm{n}, \gamma)$ is capable of generating $\gamma$-rays with energies in excess of 8-10 MeV because the neutron can be captured. These capture $\gamma$-rays produce observable signals in the Gamma Reaction History (GRH) diagnostic. GRH is an ideal detector for these $\gamma$-rays since it is inherently insensitive to the high flux of low-energy photons from laser-plasma interactions, and has a temporal resolution sufficient to differentiate between photons from $14 \mathrm{MeV}$ neutrons inelastically scattering off of TARPOS materials, and lower energy neutrons capturing on the Gold in the hohlraum.

A minimum threshold GRH channel $\left(E_{\text {threshold }}=2.9 \mathrm{MeV}\right)$ will see a prompt signal $(\mathrm{t}<1$ ns) from a standard indirect drive HTD or DT NIF capsule+hohlraum dominated by nuclear interactions on materials within $5 \mathrm{~cm}$ of TCC, since the velocity of a $14 \mathrm{MeV}$ neutron is $5.1 \mathrm{~cm} / \mathrm{ns}$. The material within this radius is composed of similar masses of $\mathrm{Au}$ (13\%), $\mathrm{Al}(34 \%)$, and $\mathrm{Si}(54 \%)$ [kroll]. This choice of material is significant because while only $\mathrm{Au}$ has a significant low-energy $(\mathrm{n}, \gamma)$ cross section, the other materials can produce high energy $\gamma$-rays through $14 \mathrm{MeV}$ neutron-induced $\left(\mathrm{n}, \mathrm{n}^{\prime} \gamma\right)$. Silicon is particularly prone toward this since $\left(\mathrm{n}, \mathrm{n}^{\prime} \gamma\right)$ is the only open channel for $E_{n}<11.6 \mathrm{MeV}$.

During the first $250 \mathrm{ps}$ after burn the GRH signal will be dominated by $16.7 \mathrm{MeV}$ fusion $\gamma$-rays and interactions of the $14 \mathrm{MeV}$ neutrons with the inner $6.66 \mathrm{~mm}$ of target material, which is approximately the distance to the outside of the Si cooling rings. Once the 14 $\mathrm{MeV}$ neutrons have moved past the cooling rings the GRH signal becomes a mix of 14 $\mathrm{MeV}$ neutron interactions on the $\mathrm{Si}$ arms and the interactions of lower energy neutrons on the inner material. The situation changes again after $1100 \mathrm{ps}$ when $14 \mathrm{MeV}$ neutrons will have swept past the end of the $\mathrm{Si}$ arms and started intercepting the (mostly $\mathrm{Cu}$ ) base. Between 246 and 1100 ps the $2.9 \mathrm{MeV}$ threshold GRH signal is comprised of $14 \mathrm{MeV}$ induced ${ }^{\text {nat }} \mathrm{Si}\left(\mathrm{n}, \mathrm{n}^{\prime} \gamma\right),{ }^{27} \mathrm{Al}\left(\mathrm{n}, \mathrm{n}^{\prime} \gamma\right)$, and lower neutron energy $(50-2100 \mathrm{keV})$ capture on ${ }^{197} \mathrm{Au}$. The high-energy photon spectrum emitted from $\mathrm{Au}(\mathrm{n}, \gamma)$ that would be visible to GRH has been measured [krticka] and is known on average to be comprised of 2-3 $\gamma$-rays covering a broad energy range of $2-7 \mathrm{MeV}$. A similar $\gamma$-ray spectrum is generated by the inelastic scattering of higher energy $(2.9-14 \mathrm{MeV})$ neutrons off of $\mathrm{Si}$.

These two time limits correspond to a "window" where the GRH signal from $\mathrm{Au}(\mathrm{n}, \gamma)$ competes only with the (n,n' $\gamma$ ) of (mostly) $14 \mathrm{MeV}$ neutrons in the Si arms. The total signal for each of these components during this time window is given by: 


$$
\begin{aligned}
& N_{A u}^{\gamma}=\int_{\text {hohlraum }} \int_{246 p s}^{1100 p s} \int_{E_{v}} M_{A u}\left(\vec{r}_{A u}\right) \Phi_{v}\left(E_{v}\right) \sigma_{(n, \gamma)}^{A u}\left(E_{v}\right) d E_{v} d t d \vec{r} \\
& N_{S i}^{\gamma}=\int_{\text {Siarms }} \int_{246 p s}^{1100 p s} \int_{E_{v}} M_{S i}\left(\vec{r}_{S i}\right) \Phi_{v}\left(E_{v}\right) \sigma_{\left(n, n^{\prime} \gamma\right)}^{S i}\left(E_{v}\right) d E_{v} d t d \vec{r}
\end{aligned}
$$

Where $\Phi_{v}$ is the neutron flux, $M_{A u}$ and $M_{S i}$ are the mass distributions of the Au in the hohlraum and the $\mathrm{Si}$ in the arms respectively and $\sigma_{(\mathrm{n}, \gamma)}^{A u}$ and $\sigma_{\left(\mathrm{n}, \mathrm{n}^{\prime} \gamma\right)}^{S i}$ are the cross sections for photon production on $\mathrm{Au}$ and $\mathrm{Si}$. The flux of 2.9-14 MeV neutrons and the average $\mathrm{Si}\left(\mathrm{n}, \mathrm{n}^{\prime} \gamma\right)$ cross section is far higher than the corresponding numbers for $<2 \mathrm{MeV}$ neutrons and the $\mathrm{Au}(\mathrm{n}, \gamma)$ cross section. However, the Si arms see far less of the neutron flux than the $\mathrm{Au}$ in the hohlraum due to the smaller solid angle $\left(10^{-3-4} \mathrm{vs.} 65 \%\right.$ of $\left.4 \pi\right)$ they subtend.

A simple model has been developed for the distribution of Si and Au in the THD capsule/hohlraum assembly for the Rev. 4 NIC target engineering drawings from Kroll [kroll]. The details of this mass model us summarized in Table 1 below. The model assumes the following:

1. A simplified version of the material position/quantity for the 544 THD hohlraum assembly [kroll] is assumed where:

a. The Au in the hohlraum is assumed to be a "ring" with a $1 \mathrm{~mm} \times 1 \mathrm{~mm}$ cross section located at the equator of the hohlraum.

b. No organic epoxy mass along the Silicon arms is taken into account.

2. A smooth functional fit for the $\mathrm{Au}(\mathrm{n}, \gamma)$ cross section derived from experimental data in the CSISRS/EXFOR database is used $\left(\sigma_{A u(n, \gamma)}=(84 \mathrm{mb}) E_{n}(\mathrm{MeV})^{-0.58}\right)$.

3. A constant $750 \mathrm{mb}$ cross section for the ${ }^{\mathrm{nat}} \mathrm{Si}(\mathrm{n}, \mathrm{n} \gamma)$ is used. This is likely to be somewhat of an overestimation since the total cross section is near this size.

4. The effective temporal error bar in Figure 4 below represents the temporal "smearing" resulting from the Au mass being distributed over the entire hohlraum instead of being in an equatorial ring. 


\begin{tabular}{|c|c|c|c|c|c|}
\hline \multicolumn{6}{|c|}{ THD_544_CH_185_913_Au_184_57_D78 Target } \\
\hline & Material $\mathrm{I}$ & Mass (g) & Volume $\left(\mathrm{mm}^{3}\right)$ & \# & Total Mass (g) \\
\hline TMP Shell & $\mathrm{Al}$ & 0.085 & 31.639 & 2 & 0.171 \\
\hline Diagnostic Band & $\mathrm{Al}$ & 0.023 & 8.490 & 1 & 0.023 \\
\hline Hohlraum & $A u$ & 0.065 & 3.374 & 2 & 0.130 \\
\hline Capsule (CH) & $\mathrm{CH}$ & 0.003 & 2.253 & 1 & 0.003 \\
\hline LEH & $\mathrm{Au}$ & 0.017 & 0.872 & 2 & 0.034 \\
\hline Washer/Window & $\mathrm{Al}$ & 0.006 & 2.085 & 2 & 0.011 \\
\hline Total & & & & & 0.372 \\
\hline \multicolumn{2}{|c|}{ Material } & Mass (g) & Vol. $\left(\mathrm{mm}^{3}\right)$ & \# & Total Mass $(\mathrm{g})$ \\
\hline \multicolumn{2}{|c|}{ Si arms (w/in radius) } & 0.078 & 31.547 & 2 & 0.155 \\
\hline \multicolumn{2}{|c|}{ Si arms (outside radius) } & 0.243 & 103.753 & 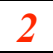 & 0.485 \\
\hline \multicolumn{2}{|c|}{ Shield Al, inner (w/in radius) } & s) 0.006 & 2.112 & 2 & 0.011 \\
\hline \multicolumn{2}{|c|}{ Shield Al, inner (outside rad) } & \begin{tabular}{l|l} 
d) & 0.024 \\
\end{tabular} & 8.868 & 2 & 0.048 \\
\hline \multicolumn{2}{|c|}{ Shield Al, outer (w/in radius) } & s) 0.010 & 3.758 & 2 & 0.020 \\
\hline \multicolumn{2}{|c|}{ Shield Al, outer (outside rad) } & 1) 0.043 & 16.036 & 2 & 0.087 \\
\hline \multicolumn{2}{|l|}{ Shield Al, annular } & 0.007 & 2.726 & 2 & 0.015 \\
\hline \multicolumn{5}{|c|}{ Total mass within $6.66 \mathrm{~mm}$ radius } & 0.559 \\
\hline \multicolumn{5}{|c|}{ Total mass outside $6.66 \mathrm{~mm}$ radius } & 0.635 \\
\hline \multicolumn{5}{|l|}{ Total target mass } & 1.194 \\
\hline
\end{tabular}

Table 1: Constituents and mass of the materials comprising the 544 THD hohlraum used in the model described above. The materials responsible for the majority of the signal from 250 ps-1000 ps after "bang" are in red bold italics. While the Al TMP shell contains significant material it is not included. This is because the $14 \mathrm{MeV}$ neutron interact with it within 250 ps of "bang" and the $\mathrm{Al}(\mathrm{n}, \gamma)$ cross section at lower neutron energies is much smaller (X100-X1000) than $\mathrm{Au}(\mathrm{n}, \gamma)$.

Figure 4 below shows the total $\gamma$-ray yield as a function of time from neutron capture on the Gold in the hohlraum, and inelastic scattering of $14 \mathrm{MeV}$ neutrons on the Silicon cooling rings and arms for the $1.1 \mathrm{~kJ}$ yield $\mathrm{HT}(+0.5 \% \mathrm{D})$ spectrum showed in Figure 1 above. An average horizontal error bar in the figure represents the effect of the Gold being located throughout the entire hohlraum, as opposed to an equatorial ring. These results are also listed below in numerical form in Table 2 below for several time bins within the 246-1100 ps window. It should be noted that for times $>1100$ ps the $14 \mathrm{MeV}$ neutrons will have swept past the Silicon arms and started interacting with more massive components in the TARPOS. 


\begin{tabular}{|c|c|c|c|}
\hline Time bin $(\mathrm{ps})$ & \# of Au $\gamma$-rays & \# of Si $\gamma$-rays & $R_{\text {Au/Si }}$ \\
\hline \hline $246-1100$ & $1.58 \times 10^{10}$ & $1.09 \times 10^{10}$ & 1.46 \\
\hline $400-1100$ & $1.25 \times 10^{10}$ & $5.38 \times 10^{9}$ & 2.32 \\
\hline $600-1100$ & $1.03 \times 10^{10}$ & $2.44 \times 10^{9}$ & 4.21 \\
\hline $800-1100$ & $9.35 \times 10^{9}$ & $1.04 \times 10^{9}$ & 9.02 \\
\hline
\end{tabular}

Table 2: \# of $\gamma$-rays generated from a lower time limit until 1100 ps post-shot from neutron capture on the Au hohlraum and inelastic scattering off of the $\mathrm{Si}$ arms. The ratio of Au to $\mathrm{Si} \gamma$-rays, which is effectively signal-to-noise, is in the last column.

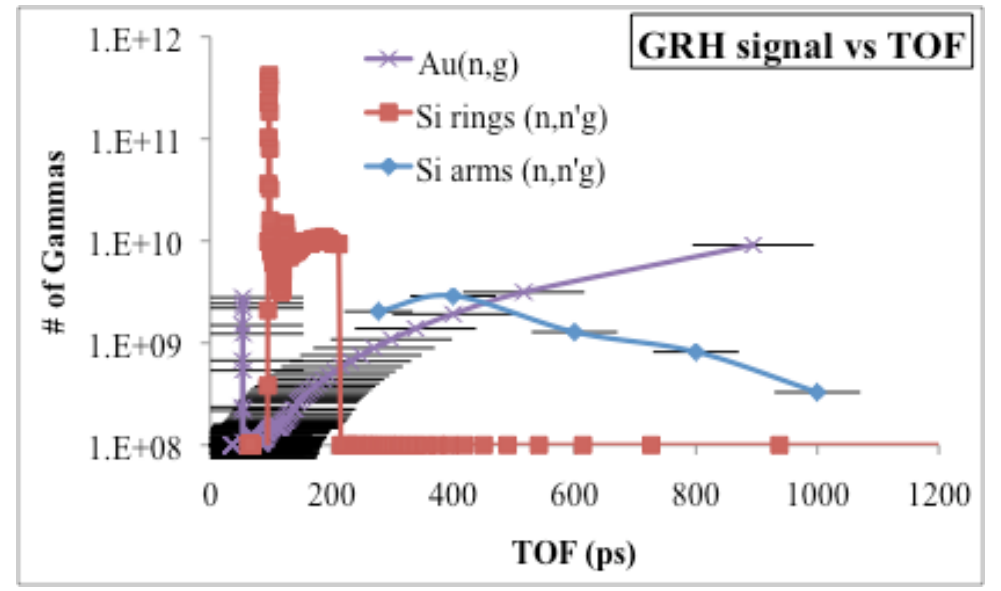

Figure 4: The number of $\gamma$-rays produced vs. time from interaction between the $14 \mathrm{MeV}$ neutrons and the Silicon in the cooling rings and arms (red and purple) and lower energy neutrons capturing on the Gold in the hohlraum.

As can be seen the Au capture "signal" to Si inelastic scatter "noise" ratio becomes increasingly favorable over time, becoming $>1$ for $t>500$ ps. It should be noted that the signal from capture on Gold increases linearly with the amount of Gold present.

A number of steps remain in order to quantify the ability of this approach to determine cold fuel $\rho R$ :

1. The spectrum of $\gamma$-rays from the decay of excited ${ }^{198} \mathrm{Au}$ resulting from neutron capture and ${ }^{28} \mathrm{Si}$ from inelastic scattering must be determined. This data is in the existing database, but recent measurements indicate that it is highly suspect (see last section below).

2. These $\gamma$-ray spectra must be convolved with the response function of the appropriate GRH channel.

If it is determined that there is insufficient signal from Au capture, additional strength can be provided through the introduction of a small diagnostic "ring" near the equator of the hohlraum. This ring could be comprised of $\mathrm{Au}$ or another favorable material. 


\section{The sensitivity of GRH as a $\rho R_{f u e l}$ diagnostic}

The low energy neutrons that give rise to the neutron capture signal from 246-1100 ps from the $\mathrm{Au}$ in the hohlraum come from scattering of low-energy neutrons in the cold dense fuel layer. These down-scattered neutrons form the basis of the Xenon radchem $\rho R$ diagnostic in that ${ }^{124} \mathrm{Xe}(\mathrm{n}, \gamma)$ has been shown in simulations to scale linearly with both fuel $\rho R$ and the number of down-scattered neutrons from 6-10 MeV. It is therefore reasonable to assume that the GRH signal in this time window would also linearly scale with fuel $\rho R$. To test this assumption the simple geometrical model described above in eqn. 1 above was convolved with two identical yield (251 J) HT(+0.5\%D) neutron spectra where inelastic scattering on hydrogen isotopes in the cold fuel was "turned on" and turned off". The spectra are shown in Figure 4 below. The ratio of the $\gamma$-ray signal from captures on the $\mathrm{Au}$ is shown in the inset.
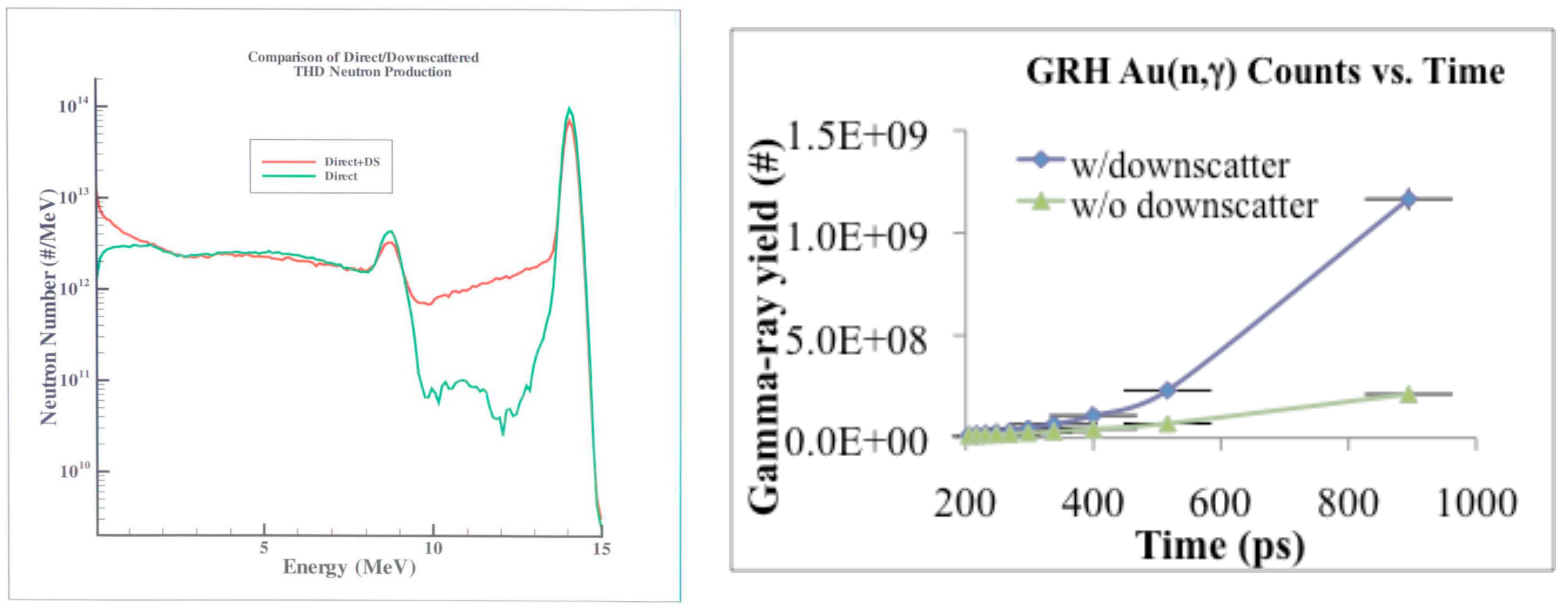

Figure 4: (Left) Neutron spectrum from a $251 \mathrm{~J} \rho R_{\text {mac }}=1.2 \mathrm{~g} / \mathrm{cm}^{2} \mathrm{HT}(+0.5 \% \mathrm{D})$ capsule with inelastic scattering on hydrogen isotopes turned off (green) and on (red). (Right) Calculated $\gamma$-ray yield from neutron capture on the $\mathrm{Au}$ in the hohlraum as a function of time. Note that the lower yield as compared to the $1.1 \mathrm{~kJ}$ case considered above in Table 2 is due in large part to the lower yield. The $\mathrm{x}$-error bar arises from the convolution of the burn time and the GRH detector temporal response.

The ratio of the number of $\gamma$-rays from capture on the Au hohlraum for the two cases shown above from $800-1100 \mathrm{ps}$ is $\approx 4$. The signal-to-noise ratio in this same time bin (from Table 2) is 9:1. This indicates that this diagnostic could potentially be used to measure $\rho R_{\text {mac }}=$ over a range from $0.3-1.2 \mathrm{~g} / \mathrm{cm}^{2}$. This diagnostic has significantly different systematic errors from the other planned $\rho R_{\text {mac }}$ diagnostic, such as $20 \mathrm{~m}$ neutron time-of-flight and the magnetic recoil spectrometer, and could provide valuable additional information to the ignition campaign.

\section{Implementation at NIF}

The best approach is to start in a "ride-along" mode using at least one low-energy (2.9 $\mathrm{MeV}$ threshold) GRH channel to detect capture $\gamma$-rays. A comparison between shots where the hohlraum contains different material (e.g., Uranium vs. Gold) would allow a 
crosscheck of the method due to the well-known cross sections for $\gamma$-ray production on both materials.

In a latter implementation we would like to introduce a thin $(<2 \mathrm{~mm})$ band of different materials, starting with additional Gold, near the equator of the hohlraum. This band could have "cut-outs" to allow for visual access for fuel quantification and X-ray diagnostics. A band would have the advantage of decreasing temporal "smearing" from low-energy neutrons arriving at different parts of the hohlraum at different times. The addition of these bands will be cleared through the NIF change control board process with specific attention paid to any potential issue of additional debris generation.

\section{References:}

[Kroll] Jeremy Kroll, Integrated Target Systems Team Private Comm. (2010) 


\section{Task II: Documentation of potential late-time $\gamma$-ray based mix measurements arising from $(\alpha, n \gamma)$ reactions in the hot spot}

Early simulation efforts indicated that $\alpha$ - and $D$ - and p-induced nuclear reactions on material implanted in the inner layer of the ablator are extremely sensitive indicators of ablator-fuel mix. This can be attributed to the limited range of charged particles in the ablator material. The numbers of nuclei produced in these reactions is typically quite modest $\left(\leq 10^{-6}\right.$ products/DT reaction). A number of these reactions were considered as a part of the radchem diagnostic, including ${ }^{18} \mathrm{O}(\alpha, \mathrm{n}){ }^{21} \mathrm{Ne},{ }^{127} \mathrm{I}(\mathrm{D}, 2 \mathrm{n}){ }^{127} \mathrm{Xe} /{ }^{127} \mathrm{I}(\mathrm{p}, \mathrm{n}){ }^{127} \mathrm{Xe}$ and ${ }^{79} \mathrm{Br}(\mathrm{D}, 2 \mathrm{n}){ }^{79} \mathrm{Kr} /{ }^{79} \mathrm{Br}(\mathrm{p}, \mathrm{n}){ }^{79} \mathrm{Kr}$. The first of these suffers from significant background from stable ${ }^{21} \mathrm{Ne}$ in the chamber, while the latter two appear likely to work at higher yields. Table 3 below shows the simulated yield for a number of charged-particle reactions where $10^{14}$ atoms of the seeded nuclei (1-3 ng) were present in the ablator, the cold fuel and the hot spot.

\begin{tabular}{|c|c|c|c|c|c|}
\hline Case & Region Loaded & O18(a,n)Ne21 & Be9(a,n)C12 & C13(a,n)O16 & F19(a,n)Na22 \\
\hline DT Failure & Hot Spot & $9.3350 \mathrm{E}+10$ & $1.8416 \mathrm{E}+10$ & $6.7526 \mathrm{E}+09$ & $4.0656 \mathrm{E}+06$ \\
\hline $397 \mathrm{~kJ}$ & Cold Fuel & $1.4740 \mathrm{E}+10$ & $3.1634 \mathrm{E}+09$ & $1.1587 \mathrm{E}+09$ & $6.2068 \mathrm{E}+05$ \\
& Inner ablator & $4.6300 \mathrm{E}+08$ & $1.0570 \mathrm{E}+08$ & $3.8609 \mathrm{E}+07$ & $1.9841 \mathrm{E}+04$ \\
& & & & & \\
& & & & & \\
THD Failure & Hot Spot & $3.5126 \mathrm{E}+07$ & $7.9850 \mathrm{E}+06$ & $2.9760 \mathrm{E}+06$ & $1.3943 \mathrm{E}+03$ \\
251 J & Cold Fuel & $3.6307 \mathrm{E}+06$ & $8.9856 \mathrm{E}+05$ & $3.3197 \mathrm{E}+05$ & $1.3999 \mathrm{E}+02$ \\
& Inner ablator & $1.1400 \mathrm{E}+03$ & $1.7405 \mathrm{E}+02$ & $5.4648 \mathrm{E}+01$ & $0.0000 \mathrm{E}+00$ \\
\hline
\end{tabular}

Table 3: Calculated reaction rates for $10^{14}$ atoms of ${ }^{18} \mathrm{O},{ }^{9} \mathrm{Be},{ }^{13} \mathrm{C}$ and ${ }^{19} \mathrm{~F}$ introduced into the hot spot, cold fuel and inner ablator from Cerjan for two cases, a $251 \mathrm{~K}$ THD failure and a $397 \mathrm{~kJ}$ DT failure.

These charged-particle nuclear reactions often produce nuclei that emit a significant amount of energy in the form of $\gamma$-rays following particle evaporation. Here we will examine the production of these $\gamma$-rays and discuss the possibility of measuring them using either the GRH or X-ray-based diagnostics planned for NIF. GRH will be considered first.

\section{GRH as a mix diagnostic}

We will start by considering the production of prompt ( $\mathrm{t} \leq 250 \mathrm{ps}$ ) high-energy $\gamma$-rays from $\alpha$-induced reactions as a mix diagnostic. During this time the GRH-observable $\gamma$-ray flux is dominated by the $16.7 \mathrm{MeV} \gamma$-ray from the $\mathrm{D}(\mathrm{T}, \mathrm{n}) \alpha$ reaction and, in the case of a plastic capsule, the $4.44 \mathrm{MeV} \gamma$-ray from ${ }^{12} \mathrm{C}\left(\mathrm{n}, \mathrm{n}^{\prime} \gamma\right)$. The fusion $\gamma$-ray has a branching ratio of approximately $4 \times 10^{-5}$, and simulations from L. Dauffy [dauffy] indicate an effective branching ratio for the ${ }^{12} \mathrm{C}$ of $\approx 5 \times 10^{-3}$. Measurements of the response of GRH at $4.4 \mathrm{MeV}$ (for a $3 \mathrm{MeV}$ threshold channel) compared to the $16.7 \mathrm{MeV}$ shows a relative efficiency $\approx 30$ times smaller. This makes the ${ }^{12} \mathrm{C}$ signal roughly 5 times larger than the fusion $\gamma$-ray. Experimental work at LLE corroborates these numbers. 
These prompt 4.4 and $16.7 \mathrm{MeV} \gamma$-ray yields place a significant constraint on any $\gamma$-raybased mix diagnostic utilizing GRH. A systematic survey of high-energy $\gamma$-rays produced by $\alpha$-induced reactions resulted in only one potential candidate; ${ }^{15} \mathrm{~N}$. The only $\alpha$-induced reaction that can produce this nucleus is ${ }^{11} \mathrm{~B}(\alpha, \gamma){ }^{15} \mathrm{~N}$. However, the cross section for this reaction is extremely small $(\leq \mu \mathrm{b})$ making it unsuitable for diagnostic purposes.

Another potential method for producing ${ }^{15} \mathrm{~N}$ is through $14 \mathrm{MeV}$ proton induced reactions from the $\mathrm{D}\left({ }^{3} \mathrm{He}, \mathrm{p}\right)^{4} \mathrm{He}$ reaction on trace Helium contamination in the capsule from tritium $\beta$-decay. Given the smaller abundance of Helium this proton flux is typically 100 times smaller than the DT neutron flux. These energetic protons could potentially produce ${ }^{15} \mathrm{~N}$ via the ${ }^{18} \mathrm{O}(\mathrm{p}, \alpha)$ reaction. While the $\mathrm{Q}$-value to the ground state for this reaction is +3.95 $\mathrm{MeV}$, the energy required to produce the $\gamma$-ray "signal" at $6.3 \mathrm{MeV}$ is a minimum of $\approx-$ 2.6 MeV. The $\gamma$-ray production cross sections for this reaction have been measured at 41 $\mathrm{MeV}$ [manitoba] and parameterized using the DWUCK direct reaction model, so we can infer the production at $E_{p}=14 \mathrm{MeV}$ to be no larger than $20 \mathrm{mb}$ (within a factor of 2).

If we scale the cross sections for ${ }^{18} \mathrm{O}(\alpha, \mathrm{n})$ reaction listed in Table 3 by this smaller high energy $\gamma$-ray production cross section for ${ }^{18} \mathrm{O}(\mathrm{p}, \alpha)$ reaction, and by the smaller $14 \mathrm{MeV}$ proton flux we can obtain an estimated $\gamma$-ray production cross section $10^{6}$ times smaller than that produced by inelastic scattering of $14 \mathrm{MeV}$ neutrons on the ${ }^{12} \mathrm{C}$ in the plastic ablator. Since both of these $\gamma$-rays are observed in the same GRH channel, which also sees the intense $16.7 \mathrm{MeV}$ fusion $\gamma$-ray, it is extremely unlikely that a good signal could be extracted over the prompt $\gamma$-ray background.

The situation is significantly different for a Beryllium capsule. In this case there is very little competition from the ${ }^{12} \mathrm{C}\left(\mathrm{n}, \mathrm{n}^{\prime} \gamma\right)$, and the possibility exists to use the ${ }^{9} \mathrm{Be}(\alpha, \mathrm{n} \gamma)^{12} \mathrm{C}$ reaction to measure mix. The only issue here is whether background from the DT fusion $\gamma$-ray will dominate the prompt signal. The results shown for this reaction in Table 3 above indicate an effective $\gamma$-ray production of approximately $10^{-4}$, making it comparable to the production of the $16.7 \mathrm{MeV}$ fusion $\gamma$-ray. However, the lower sensitivity for GRH at $4.4 \mathrm{MeV}$ makes this a difficult experiment at best.

In short, there is no clear way that mix can be diagnosed using GRH at NIF in plastic capsules, but there is some possibility of this approach working for a low-Carbon content capsule, such as Beryllium.

\section{Using late-time $(200 \mathrm{~ns}<\mathrm{t}<5 \mu \mathrm{s}) \gamma$-rays as a mix diagnostic}

During the preparation of this report a new potential source of mix information was considered; the production of $\gamma$-rays from long-lived nuclear states formed in chargedparticle reactions on seed nuclei in the NIF capsule. Figure 5 below shows where the current set of photon diagnostics at NIF cover in the energy-time phase space. 
Simulations by Eder et al., [eder] has shown that the vast majority of the laser-plasma induced photon background in an indirect drive NIF shot is "over" by $\approx 90$ ns after "bang". In addition, these simulations show that the majority of the hohlraum material, in which all capsule debris would be "caught" moves at velocities much less than 1.5 $\mathrm{cm} / \mu \mathrm{s}$, meaning that the debris is still within a short distance of TCC for several $\mu \mathrm{s}$ after the shot. In addition, the $14 \mathrm{MeV}$ neutrons reach the target chamber wall $\approx 100$ ns after the shot and generate a large $\gamma$-ray background that returns to TCC approximately $17 \mathrm{~ns}$ later. Taken together, these results indicate there is a "quiet window of opportunity" from approximately $200 \mathrm{~ns}$ to $5+\mu \mathrm{s}$ where the majority of the hohlraum material is still within a narrow range of TCC and the background from the laser-induced implosion is greatly decreased.

This time range is significant because many low-lying excited nuclear states that decay via $\gamma$-ray emission have lifetimes in this time range. These isomers therefore offer the possibility of serving as nuclear diagnostics if the appropriate "target" nucleus is loaded into the capsule.

One example that appears particularly compelling is the production of the $583 \mathrm{keV}$ isomer in ${ }^{22} \mathrm{Na}$ via the ${ }^{19} \mathrm{~F}(\alpha, \mathrm{n})$ reaction. This state has a lifetime of $243 \mathrm{~ns}$ and has a "reasonable" cross section for activation via exposure to the $3.5 \mathrm{MeV} \alpha$ 's from fusion. Table 3 above shows the production of this nucleus.

The ideal diagnostic for the observation of photons from these isomeric nuclear transitions would have large solid angle, be "switched off" until 100-200 ns after implosion, and would provide time- (and preferably energy-) resolved data. Simple designs using neutron-insensitive inorganic scintillators, such as $\mathrm{NaI}$ or CsI, close to TCC could be considered.

Late-time simulations of $\gamma$-ray production are required in order to fully explore the possibility of such a nuclear isomeric $\gamma$-ray diagnostic. However, the model of choice used for these simulations, MCNP, lacks a proper treatment of nuclear isomerism. As a result MCNP outputs would have to be "post-processed" in some way to provide an appropriate background measurement.

It is the estimate of the team that a complete initial treatment of such an isomeric $\gamma$-ray diagnostic for mix, or other important quantities, would require a minimum effort level of $2-3$ FTE for $\approx 2$ months to be carried out. This could be addressed in more detail in a subsequent proposal. 


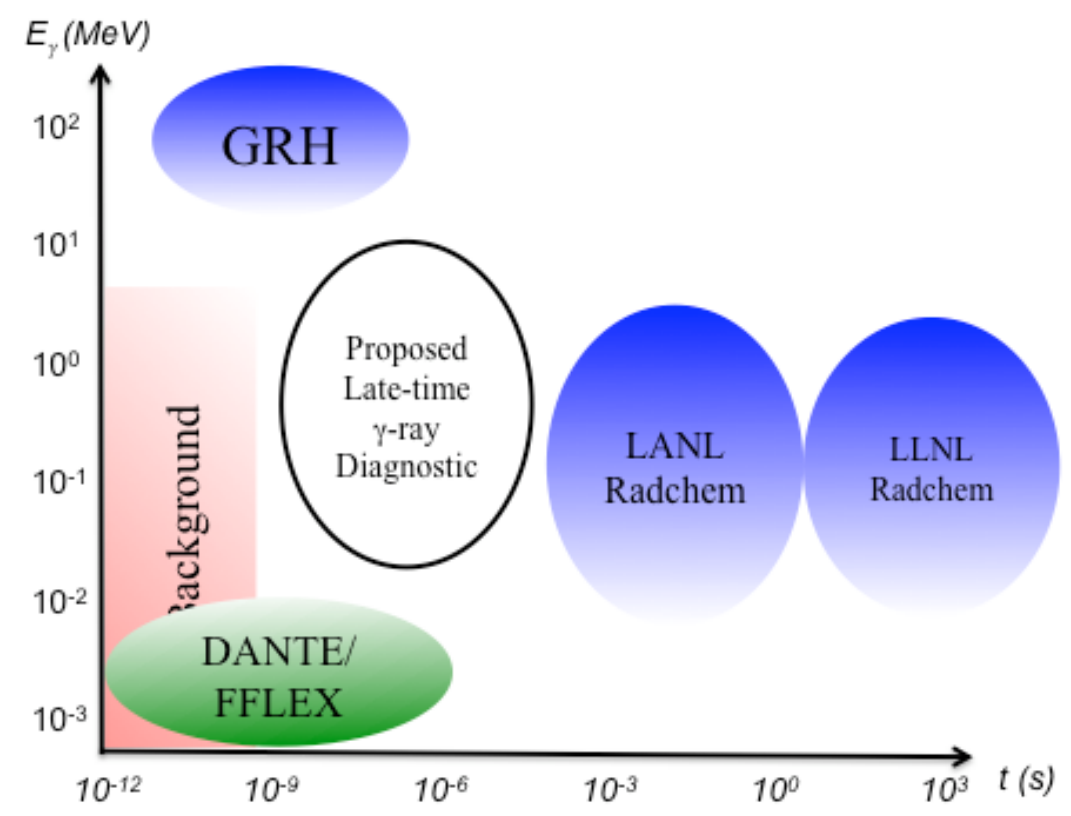

Figure 5: Schematic of existing and proposed photon diagnostics at NIF

\section{References:}

[Manitoba] The ${ }^{18} \mathrm{O}(\mathrm{p}, \alpha)^{15} \mathrm{~N}$ reaction at $40.9 \mathrm{MeV}$. J.R. Campbell, O.A. Abouzeid, W.R. Falk, R. Abeg, SK. Datta and S.P. Kwan. Nuclear Physics A467 (1987) 205-214 North-Holland, Amsterdam.

[Dauffy] Lucile Dauffy, private communications.

[eder] Late-time simulation of National Ignition Facility Hohlraums, D.C. Eder, O.S. Jones, M.M. Marinak, M.T. Tobin, B.J. MacGowan. September 21, 2004 submitted to Nuclear Fusion 2004 (UCRL-JRNL-206693). 


\section{Task III: Assessment of Nuclear Data for use in mix and $\rho R$ diagnostics}

The diagnostics discussed above have varying degrees of dependence on underlying nuclear data regarding $\gamma$-ray production in neutron- and charged-particle induced reactions. In some cases, where ratios of $\gamma$-rays can be considered, the dependence is limited. However, in the case where either the absolute $\gamma$-ray production cross section or the spectral distribution are needed, the contents of standing databases are highly suspect.

The magnitudes of the uncertainties are hard to gauge. However, recent results from LLE [cite-Kim poster] highlight these uncertainties for the production of high-energy $\gamma$-rays for a number of important low-mass nuclei. In these experiments "pucks" of $\mathrm{Al}, \mathrm{Si}, \mathrm{SiO}_{2}$ and $\mathrm{Cu}$ were placed $5.8 \mathrm{~cm}$ from TCC at LLE, and the $\gamma$-ray production from both the $\mathrm{D}+\mathrm{T}$ reaction and $(\mathrm{n}, \mathrm{x})$ on the puck material were observed in a GCD with a $5.3 \mathrm{MeV}$ threshold. These $\gamma$-ray production rates were also calculated using MCNP. The ratio of the measured to simulated $\gamma$-ray yields are shown in Figure 6 below.
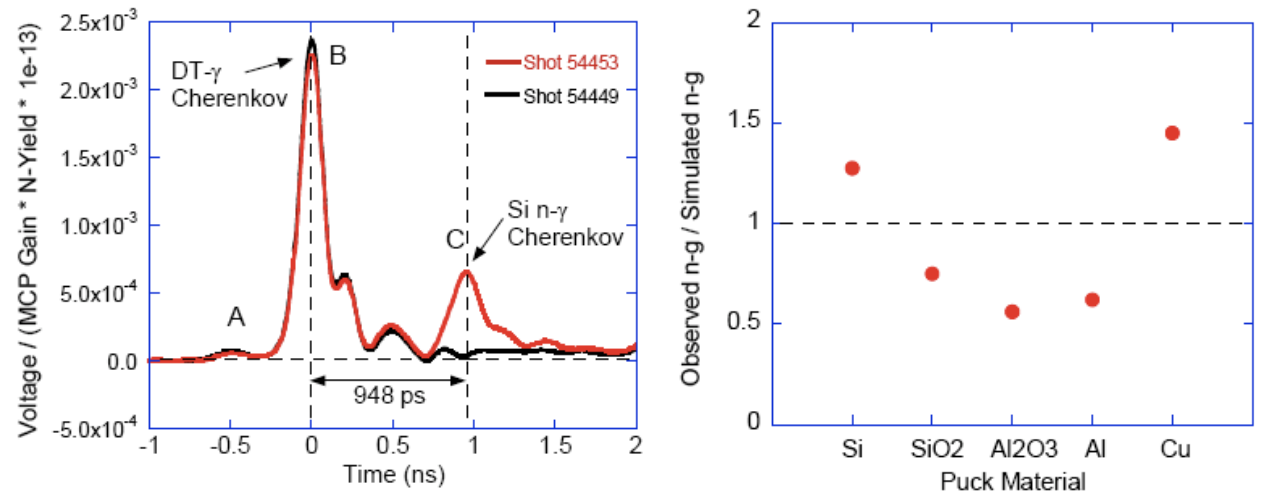

Figure 6: Results from puck irradiation experiments at LLE [cite-Kim_poster]. The left side shows the scope trace from a GCD for shots with (red) and without (black) a Silicon puck present. The right side shows the ratio of the observedto-simulated $\gamma$-ray yield.

The situation for the production of lower-energy isomeric (200 ns-5 $\mu \mathrm{s}$ ) $\gamma$-rays is harder to quantify. While there is exhaustive information regarding the characteristics of these states (including lifetime, decay energy, spin/parity etc.) this data is usually from light- and heavy-ion induced reactions, which could populate these states in significantly different proportion than $14 \mathrm{MeV}$ neutrons or $3.5 \mathrm{MeV} \alpha$-particles. A measurement of the isomeric $\gamma$-ray production cross section for these states would be fairly straightforward and would require no more than 2-3 months effort by a 2 FTE team. 


\section{Appendix A: Neutron Capture Measurements using pure deuterium-filled capsules}

A pure deuterium filled capsule creates a neutron spectrum that is highly suited for neutron capture cross section measurements due to the near-absence of $14 \mathrm{MeV}$ neutrons from the $\mathrm{D}+\mathrm{T}$ reaction. Primary neutrons from the $\mathrm{D}+\mathrm{D}$ reaction are $2.45 \mathrm{MeV}$ and are incapable of producing $\gamma$-rays of sufficiently high energy via inelastic scattering to create a signal in the GRH diagnostic.

The number of the $\gamma$-rays produced from neutron capture on $10^{16}$ nuclei located in the inner layer of the ablator yields a number close to that seen for the more massive hohlraum $\left(\approx 10^{21} \mathrm{Au}\right.$ atoms $)$ due to the increased areal density of material in the ablator arising from the capsule compression. If we assume compression from $1 \mathrm{~mm}$ to $30 \mu \mathrm{m}$ in radius without mass loss then we obtain an increase in areal density of $(1 / 0.03)^{2} \approx 10^{3}$. Table 5 below shows the $(n, \gamma)$ cross sections and number of $\gamma$-rays formed at both peaks in the neutron energy spectrum $(5-100 \mathrm{keV}$ and $2450 \mathrm{keV}$ respectively) for several potentially interesting candidate nuclei. Figure 5 below shoes the number of g-rays produced as a function of time for a capsule with $10^{16}$ atoms of ${ }^{74} \mathrm{Ge}$ and a Au hohlraum.

\begin{tabular}{|c|c|c|c|}
\hline Nucleus & $(\mathrm{n}, \gamma)$ Cross Section at $50 \& 2450 \mathrm{keV}$ & \# of $(\mathrm{n}, \gamma)$ \\
\hline${ }^{74} \mathrm{Ge}$ & $254 \mathrm{mb}$ & $11.2 \mathrm{mb}$ & $3 \times 10^{8}$ \\
\hline${ }^{124} \mathrm{Xe}$ & $1.38 \mathrm{~b}$ & $460 \mathrm{mb}$ & $1.8 \times 10^{9}$ \\
\hline${ }^{169} \mathrm{gm}$ & $1.44 \mathrm{~b}$ & $18.3 \mathrm{mb}$ & $1.34 \times 10^{9}$ \\
\hline${ }^{169 \mathrm{~m}} \mathrm{Tm}$ & $2.33 \mathrm{~b}$ & $19.9 \mathrm{mb}$ & $2.02 \times 10^{8}$ \\
\hline
\end{tabular}

Table 4: $(\mathrm{n}, \gamma)$ cross sections and number of reaction products (assuming $10^{16}$ nuclei loaded at the ice-ablator interface) for 4 potentially interesting nuclei. Cross sections are taken from ENDF-B7 for ${ }^{74} \mathrm{Ge}$ and ${ }^{124} \mathrm{Xe}$ and from the recent paper from Kawano et al. [cite-kawano] for the two states in ${ }^{169} \mathrm{Tm}$.

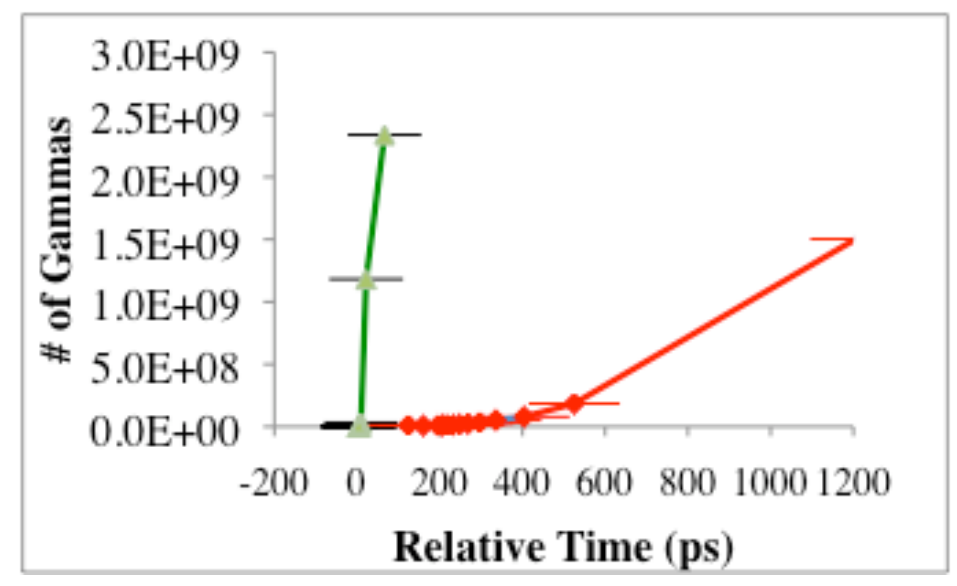

Figure 5: GRH signal from a capsule loaded with $10^{16}$ of ${ }^{74} \mathrm{Ge}$ (green) and the $\mathrm{Au}$ in the hohlraum (red). The slight incline in the prompt peak now arises from very low energy $(<20 \mathrm{keV})$ neutrons interacting with the ablator material. 